\title{
LA PRÁCTICA FILOSÓFICA COMO UNA ACTIVIDAD SOCIALMENTE COMPROMETIDA:
}

\author{
Diálogo filosófico y desarrollo de la autonomía en un contexto de reeducación de \\ menores infractores
}

Víctor Andrés Rojas

Uniminuto

\section{Resumen}

El acercamiento de niños a la filosofía en ciertos sectores de educación no formal y en el trabajo con poblaciones marginadas, entendido primero como programa educativo y después como proyecto de educación filosófica, ha llevado a la necesaria tarea de identificar los rasgos fundamentales que hacen de esta propuesta un proyecto adecuado para la formación de jóvenes que se encuentran en proceso de reeducación. Tal es el caso de las prácticas desarrolladas a partir del 2006 en el Centro Educativo Amigoniano Escuela de Trabajo El Redentor, con algunos grupos y comunidades terapéuticas.

Palabras claves: filosofía para niños, educación no formal, filosofía y población marginada.

\begin{abstract}
Philosophical practice as a socially committed activity: philosophical dialogue and thedevelopment of autonomy in a context of reeducation of minor infractors.

Initiating the contact of children with philosophy in certain sectors of non-formal education and work with marginalized populations, understood in the first hand as educational program and secondly as philosophical education project, has led to the necessary task of identifying the fundamental qualities that make this proposal an adequate project for the formation of youths in the process of reeducation. Such is the case of the teaching practicum developed from 2006 onward at the Amigoniano Educational Center El Redentor Work School, with some groups and therapeutic communities.
\end{abstract}

Key words: philosophy for children, non-formal education, philosophy and marginalized population

\section{La educación reflexiva en el proceso de enseñanza-aprendizaje}

En el presente trabajo se pretende ampliar la reflexión sobre algunas características de la filosofía para niños $(\mathrm{FpN})$ en relación con la práctica pedagógica realizada en una institución reeducativa, señalando de manera particular las categorías de diálogo y autonomía como elementos emergentes y necesarios en la comunidad de indagación. Dicha experiencia en el Centro El Redentor, significó para los practicantes una oportunidad para identificar los límites y alcances de Filosofía para Niños (FpN) en esta población y significó para la comunidad la posibilidad de dialogar con otras propuestas 
que permiten complementar la formación en habilidades argumentativas, cognitivas y sociales.

Uno de los elementos necesarios de revisar a la hora de proponer FpN en la dinámica formativa de una institución como El Redentor consiste en identificar las características del sistema pedagógico que desarrolla la institución y la relación a nivel educativo que tendría con los cambios que pueden sugerir a partir del desarrollo de FpN. Para ésto es importante tener en cuenta que $\mathrm{FpN}$, más allá de pretender implementar un currículo integrado por novelas filosóficas, hoy se presenta como un proyecto de educación filosófica que, si bien puede aprovechar los textos clásicos del programa, logra aportar en la formación de personas críticas en una sociedad como la nuestra en la que el tema de la participación y la democracia requieren de elementos como el diálogo y la autonomía para su desarrollo. De esta manera, quisiera señalar a continuación algunos elementos que sugiere la práctica filosófica en el momento de pensar en FpN como una propuesta de educación filosófica; en primer lugar teniendo en cuenta que esto contribuirá a la estructuración de un nuevo modelo educativo en el que se estimule fundamentalmente el pensamiento crítico.

A mi manera de ver, la gran contribución de Lipman es el desarrollo de una conciencia reflexiva en los participantes de una comunidad de indagación, logrando de esta manera plantear la educación no como la continuación de un modelo estándar en el que los involucrados sean tomados como sujetos pasivos que hacen preguntas y piensan cuando aprenden lo que se les está enseñando, sino que estos piensen de manera conjunta en una comunidad de indagación conformada por personas críticas ${ }^{1}$.

Incorporar estas prácticas reflexivas en una institución implicaría pasar de una educación basada en la transmisión de conocimientos de aquellos que saben a aquellos que no saben, a una educación resultado de la participación en una comunidad de diálogo. Esto implica moverse de una concepción de conocimiento sobre el mundo precisa, inequívoca y determinada, a un conocimiento dividido en disciplinas que no se sobreponen entre sí y que juntas abarcan los problemas propios del conocer; de profesores autoritarios en el proceso educativo que piensan que los estudiantes deben conocer lo que ellos conocen, a profesores que admiten que pueden estar equivocados y asumen el papel de guías que animan a los estudiantes a pensar sobre el mundo de forma abierta y dinámica; de estudiantes que adquieren el conocimiento mediante la absorción de datos e información, a estudiantes reflexivos, pensantes y que incrementan su capacidad de razonabilidad y de juicio.

En el modelo reflexivo se piensa que las disciplinas en las cuales se generan procesos de indagación pueden yuxtaponerse entre ellas, no siendo exhaustivas en relación con su respectiva área de conocimiento. En conclusión, lo importante en este caso no es la adquisición de información, sino la indagación de las relaciones que existen en la materia que se investiga.

El modelo educativo desarrollado en el centro El Redentor se enmarca en una propuesta de formación integral que aboga por el proceso de un sujeto capaz de insertarse en un contexto social, teniendo en cuenta que ha sido afectado por problemas de orden social

\footnotetext{
${ }^{1}$ Cfr. Matthew Lipman, Pensamiento complejo y educación. Madrid: De la Torre, 1997. p. 30.
} 
que lo han llevado a delinquir en distintos órdenes. La propuesta de esta institución supone un proceso terapéutico en el que desde un tiempo de convivencia los jóvenes reciben una atención institucional a través de la ejecución y evaluación de programas de formación espiritual, axiológica, social, cultural, cívica, física, afectiva, recreativa, deportiva, artística, educación formal, no formal e informal, todo enfocado al mejoramiento de la calidad de vida y a la convivencia social ${ }^{2}$.

Dicho modelo educativo podría complementarse con el propuesto por FpN en lo que se refiere a una formación interesada en valorar las capacidades del individuo y fomentar su crecimiento integral. Sin embargo, en la práctica misma y en el intento de desarrollo de la comunidad de indagación se identificó como un principal obstáculo el carácter disciplinar de la formación que muchas veces requiere del elemento sanción y del control terapéutico. El acercamiento a esta realidad desde las prácticas profesionales generó la necesidad de fomentar un diálogo entre la práctica de $\mathrm{FpN}$ y la pedagogía de la institución, a fin de identificar la manera más adecuada de plantear la comunidad de indagación sin descuidar elementos fundamentales como la espontaneidad en las sesiones y la libertad en la participación y el respeto en la corrección. Al respecto quisiera citar la reflexión que realizan algunos de los estudiantes sobre su experiencia de prácticas en El Redentor. Ellos pudieron evidenciar las dificultades para iniciar una práctica espontánea y abierta.

La cuestión fue que ellos, al hacer el grupo, reaccionaron como la vez anterior, con actitud muy negativa y de aburrimiento, no querían hablar. Intentamos animarlos a dibujar, pero antes se les pidió que escribieran su nombre, muchos no saben escribir, o apenas deletrear su nombre y no completo. Con el dibujo y lo que decían saltó a nuestros ojos la verdad. Ellos al hablar de su vida diaria no hacían sino preguntarse entre ellos cómo se sentían, o simplemente para decir que la institución era muy buena, que les gustaba las clases de la institución, que se sentían bien, hubo un momento en que se tocó el tema de si eran libres o no y, al principio alguien intentó decir que no, pero al momento lo corrigió o simplemente se quedaban callados. Ya cuando muchos se estaban despidiendo... tuve la oportunidad de que entre abrazo y abrazo me dijeran lo que realmente pasaba. Simplemente el valor de la palabra en este lugar tiene otros trasfondos, ellos quieren irse de ese lugar, la mejor manera es seguir el juego de la institución, sólo dicen lo que la institución quiere escuchar, responden lo que les encaja en ese discurso. La desconfianza de los chicos no sólo está con los representantes de la autoridad, entre ellos se da. Así me lo han dado a entender con el decirme "esto es una cárcel", "yo me quiero ir pronto", "aquí no se puede hablar, profe, porque nos joden $^{3}$.

Ellos siempre pensaban, y se sentía en el ambiente la preocupación porque se le contara algo al educador y ahí se acababa la tranquilidad. Por este motivo también sirvió realizar actividades no sólo de FpN, sino actividades lúdicas fuera del aula, jugar ponchados, fútbol, eso fue bastante importante y fue necesario para poder superar el miedo y el temor que sentían, tanto que a veces preferían alejarse y ésto se presentaba cuando estaban los educadores, pues algunos muchachos se intimidaban con los educadores y otros estaban muy tranquilos ante su presencia ${ }^{4}$.

\footnotetext{
${ }^{2}$ Esto puede verse en el PAI. Proyecto de Atención Institucional - Centro Educativo Amigoniano: Escuela de Trabajo el Redentor (2001-2003).

3 Nubia Villamil, Diario de campo 03, Practica Profesional III, 2006.

${ }^{4}$ Víctor Andrés Rojas, Entrevista: Lida Gil, Corporación Universitaria Minuto de Dios, 2007.
} 
En las citas anteriores se hace evidente la necesidad de diseñar estrategias para un diálogo adecuado con la institución, especialmente al tener en cuenta el interés disciplinario. Como puede verse, los estudiantes inicialmente estaban condicionados a realizar ciertas actividades por una motivación externa, muchas veces represiva, sin valorar suficientemente la autonomía.

Por su parte, FpN aboga por la generación de individuos pensantes y por la transformación de una mentalidad cerrada en el ámbito educativo que implique superar concepciones educativamente limitadas respecto al ser y quehacer de la educación y, por lo tanto, del papel de los docentes y los estudiantes. Dicha transformación puede realizarse particularmente en la práctica de la comunidad de indagación, dado que ella, desde la apertura al diálogo, fortalece aspectos pilares en el desarrollo integral del individuo, tales como la formación del juicio y la autonomía.

Así pues, conceptos como indagación, comunidad, diálogo y autonomía se constituyen en esta propuesta educativa como pilares determinantes para la formación de un proyecto basado en el desarrollo de un pensar crítico y reflexivo. Por tal razón, es importante realizar un acercamiento a dichos conceptos para así comprender claramente los alcances que tendría FpN en una institución como El Redentor.

\section{Comunidad de indagación}

La comunidad de investigación o, como también es llamada, comunidad de cuestionamiento, de indagación o de diálogo es un medio para promover el sentido de comunidad, elemento fundamental en toda sociedad democrática. Este tipo de comunidad cultiva, entre otras cosas, las habilidades de diálogo, de cuestionamiento, de investigación reflexiva y del buen juicio.

Para Lipman, siguiendo las propuestas educativas de Dewey, la educación debe buscar que los estudiantes, más que aprender las soluciones, se involucren en un proceso de investigación por sí mismos, estudiando no sólo los resultados de lo que los científicos han estudiado, sino mediante un proceso de búsqueda y acercamiento investigativo al conocimiento. En dicho proceso, los participantes de la comunidad serán también investigadores que a partir del asombro, la curiosidad, las preguntas y argumentaciones podrán formular sus propios juicios y construir significados. Esto no implica caer en cierto relativismo en el que el conocimiento sea de carácter personal, descartándose toda posibilidad de conocimientos universales. Lo que se pretende es crear una actitud investigativa de carácter crítico en los estudiantes para que haya una apropiación personal y autónoma del conocimiento y no una imposición autoritaria de saberes establecidos.

Convertir la sesión en una comunidad de investigación implica que los estudiantes se escuchen entre sí con respeto, construyendo más ideas sobre las de los demás, retándose entre sí para reforzar argumentos de opiniones poco fundadas, ayudándose mutuamente en los procesos inferenciales a partir de afirmaciones y buscando identificar los supuestos ajenos. Una comunidad de investigación intenta rastrear la indagación donde sea, aunque bordee las fronteras de las disciplinas establecidas, en un diálogo que 
pretende conformarse en lógica moviéndose indirectamente aunque su avance parece semejarse al propio pensamiento ${ }^{5}$.

Un grupo de investigación, desde esta perspectiva, es resultado del esfuerzo de toda una comunidad educativa por despertar el interés de los estudiantes para la construcción autónoma o acercamiento crítico de saberes. Es, de igual forma, resultado de un cambio en la mentalidad de los docentes, quienes han de ser los primeros interesados en promover el diálogo en sus clases. Por tal razón, si se quiere implantar este tipo de actitudes en los educandos debe iniciarse por un cambio curricular en las comunidades educativas, pues de no haber un currículo abierto y flexible es imposible favorecer el pensamiento y convertir las aulas en comunidades de investigación. Solo cuando hay un currículo abierto, en el que los conceptos son flexibles a la investigación, se logra capturar la atención de los estudiantes y propiciar una comunidad de investigación.

Por otro lado, una comunidad de investigación debe buscar ciertos cambios en la actitud de los estudiantes, pues son éstos, como ya se ha dicho, los principales protagonistas de este tipo de comunidades. Para esclarecer aún más este propósito, quisiera hacer mención a algunos postulados de Ann Margaret Sharp ${ }^{6}$, quien hace al respecto un interesante planteamiento acerca de los comportamientos que se encuentran en una comunidad de indagación: ${ }^{7}$

Comportamientos cognitivos: consisten en dar y pedir buenas razones, hacer buenas distinciones y relaciones, realizar inferencias válidas, hacer hipótesis, generalizar, dar contra-ejemplos, detectar supuestos, usar y reconocer criterios, plantear buenas preguntas, inferir consecuencias, reconocer falacias lógicas, ser relevantes, definir conceptos, buscar clarificación, hacer buenos juicios, hacer construcciones lógicas sobre las contribuciones de otros y discernir diferencias sutiles. Los alumnos, a partir de la comunidad de indagación, se vuelven más tolerantes frente a la complejidad y la ambigüedad y reconocen que la justificación de las creencias tiene sus raíces en la acción humana. La capacidad de juzgar, desarrollada en estas comunidades, está basada en un sentido cívico comunitario que es necesario para hacer juicios morales y políticos. Este tipo de juicios son intersubjetivos, pues necesitan someterse al examen de otras personas capaces de juzgar.

Otro tipo de comportamientos que se pueden encontrar en las comunidades de indagación son los comportamientos sociales, manifestados en actitudes como: escuchar a otros, apoyarse unos a otros, ampliando y corroborando sus respectivos puntos de vista, someter las opiniones de otros a la investigación crítica, dar razones para apoyar la opinión de otro aun sin estar de acuerdo, tomar en serio las ideas de otros, respondiendo y alentando a que cada uno exprese sus puntos de vista. Dentro de estos comportamientos sociales se desarrolla el llamado "pensamiento cuidadoso del otro", el cual se manifiesta en aquellas actitudes en las cuales los participantes de la comunidad son capaces de cambiar sus opiniones y prioridades en vistas a tener cuidado por el otro,

\footnotetext{
5 Lipman, Op. cit., p. 58.

${ }^{6}$ Cofundadora del programa de Filosofía para Niños. Hace parte del Centro de investigaciones de Filosofía para niños ICPC en Montclair USA. En la actualidad trabaja con Lipman en la promoción del programa de FpN.

${ }^{7}$ Cfr. Ann Margaret Sharp, "La comunidad de cuestionamiento e investigación filosófico: Educación para la democracia”. En: Qué es Filosofía para Niños, ideas y propuestas para pensar la educación. Buenos Aires: Editorial Universidad de Buenos Aires, 1997. p.177,
} 
de ser afectado por él. Este cuidado es esencial para el diálogo y para el desarrollo de la confianza; "la comunidad de investigación es aprender juntos y por ello es un ejemplo del valor que tiene la experiencia compartida. Pero en otro sentido, ilustra también las posibilidades del aprendizaje conjunto, pues unos estudiantes que hasta entonces debían aprender todo individualmente, descubren de repente que pueden apoyarse y utilizar la experiencia de los de los otros" $"$.

A continuación quiero hacer referencia a una de las experiencias de práctica en el Centro El Redentor, en la cual los estudiantes identificaron las transformaciones que implicaba la puesta en práctica de la comunidad de indagación.

La lectura del cuento generó un ambiente de reflexión en medio de la comunidad de indagación, ello empezó a arrojar los primeros resultados de lo que empezaba a ser nuestro trabajo filosófico en el grupo de juventud. Si el poder hacer que los muchachos dejaran de lado tantos prejuicios, tanta discriminación, el mismo hecho de permanecer condicionados en un lugar así, todo ello hacia que nuestro trabajo empezara a determinar cambios, el solo hecho de poder expresar libremente las opiniones sin temor de ser rechazados o recriminados, hace que el trabajo rompa con los esquemas, rompa con la tradicionalidad esquemática y permita al ser humano ser lo que verdaderamente es ${ }^{9}$.

De esta manera, se pueden observar también características psicológicas o sociopsicológicas. Son aquellas que incluyen el crecimiento del yo en relación con los otros, es decir, ponen el ego en perspectiva, controlan el egocentrismo y eventualmente se transforman a sí mismos. Los participantes evitan los largos monólogos y saben cómo dialogar unos con otros. Los docentes y participantes tienen la oportunidad de callarse para dar paso a la opinión de otros, escuchar atentamente las intervenciones y dejarse llevar por el rumbo que la comunidad investigativa va abriendo.

Con este proceso ha quedado explicado con claridad un elemento fundamental en la propuesta de Lipman, como es el de la comunidad de indagación. El siguiente paso será el de comprender otros elementos que, como puede notarse, están completamente involucrados en este proceso de investigación; estos son el diálogo y la autonomía. A continuación se mostrará su intervención en este modelo reflexivo.

\section{Diálogo y autonomía}

Es necesario aclarar estos dos conceptos para la comprensión del pensamiento crítico, analizando sus relaciones e implicaciones en el desarrollo de un modelo reflexivo.

\subsection{Autonomía}

El término autonomía viene del griego $\alpha v \tau o v o \mu o \varsigma$ formado por $\alpha v \tau o \varsigma$, autos: sí mismo, y vouo nómos: ley, el que vive según su propia ley o se gobierna por su propia ley ${ }^{10}$. La autonomía, en un sentido amplio, es entendida como la capacidad de bastarse a sí mismo para preservar la propia individualidad frente a los demás o frente a la

\footnotetext{
${ }^{8}$ Lipman, Op. cit., pp. 318-319.

9 Edwin Malagón. Diario de campo 02. Práctica profesional II. 2006.

10 Antoni Martínez Riu y Jordi Cortés Morató. Diccionario de Filosofía. Barcelona: Editorial Herder, CD-ROM, 1998.
} 
colectividad, a los que, no obstante, necesita en buena medida. El concepto de autonomía en Lipman es planteado como la capacidad de pensar por sí mismo sin seguir a ciegas las opiniones de otros. La autonomía es la que permite a un individuo realizar sus propios juicios sobre sucesos, formar una comprensión del mundo propia y construir su propia concepción sobre lo que quiere ser y el mundo en el que quiere vivir. Por tal razón, la autonomía implica una liberación de todo aquello que mediante el uso del poder condiciona una manera de pensar y por lo tanto un comportamiento y estilo de vida.

Para comprender aún más el significado de la autonomía se podría citar a Kant, quien aportó importantes herramientas a la sociedad del siglo XVIII con su noción de autonomía. Tal idea la desarrolló en la época de la Ilustración, que data de 1666 a 1789, aproximadamente, y que presenta entre sus principales características un optimismo antropológico, es decir, una confianza en el poder y una autonomía de la razón fundada en la posibilidad de emplear la razón en la actuación técnica sobre la naturaleza y en la actuación moral y política sobre la sociedad. Kant, en su ensayo ¿Qué es la Ilustración?, muestra la necesidad de construir una sociedad en la que cada uno piense por sí mismo a partir de la autonomía de la razón. Para él la ilustración es la liberación del hombre de su culpable incapacidad. Tal incapacidad significa la imposibilidad de servirse de su inteligencia sin la guía de otro. Esta incapacidad es culpable porque su causa no reside en la falta de inteligencia, sino de decisión y valor para servirse por sí mismo de ella sin la tutela de otro. El lema de la Ilustración es: "Ten valor de servirte de tu propia razón""11.

La autonomía debe permitir hacer uso de la propia razón y alejarse de los principios, fórmulas e instrumentos que han impedido su realización. Sin embargo, el concepto de autonomía en Lipman no hace referencia a un acentuado individualismo ${ }^{12}$ o a una exagerada autosuficiencia, sino a un pensar por sí mismo sin seguir las ideas o mandatos externos o definidos por agentes distintos a la razón. Es decir, la autonomía tiene que ver con un proceso de pensamiento lógico y propio. Para Lipman, este pensar de manera autónoma se realiza a partir del intercambio de ideas con otros sujetos en medio de una comunidad o grupo social. Solo a partir del intercambio de postulados respecto a una idea, de la discusión y puesta en común de argumentos es como se puede formar un mejor juicio. Tal planteamiento de Lipman ha logrado llevarse a la práctica en $E l$ Redentor a través del desarrollo de las sesiones de comunidad de indagación; en estas comunidades los participantes han logrado avanzar en lo que se refiere al diálogo libre y espontáneo y en la construcción autónoma de ideas. Dicho avance es señalado por una de las directivas de la institución, que al respecto afirma lo siguiente:

se ha trabajado sobre la comunidad de indagación, el enfoque han sido esas comunidades de diálogo para ayudarles a los muchachos a ir más allá, les ha ayudado a los muchachos a reconocerse a sí mismos con sus capacidades y limitaciones, pero ellos son capaces de construir pensamiento, son capaces de construir saber, en ese orden de ideas considero que la comunidad de indagación, o

\footnotetext{
${ }^{11}$ Emmanuel Kant, Respuesta a la pregunta ¿Qué es la Ilustración?, en Filosofía de la historia. Buenos Aires: Nova, 1064.

${ }^{12}$ Acentuado individualismo tiene que ver, según Lipman, con el pensador crítico e independiente que se caracteriza como persona cognitivamente autosuficiente, atrincherado tras el poder de invencibles argumentos.
} 
comunidad de diálogo, como lo llama Uniminuto y sus estudiantes de filosofía, nos permite profundizar en los aspectos del intercambio dialógico con el otro ${ }^{13}$.

En esta entrevista realizada por un grupo de estudiantes, el director académico de la institución señala algunos de los aportes identificados en la práctica de $\mathrm{FpN}$ con los jóvenes participantes. Sin lugar a dudas uno de los mayores aportes lo hace la comunidad de indagación al generar en los individuos alternativas de pensamiento que los llevan a repensar su situación de vida y a construir de manera cooperativa ideas y pensamientos. Esto señala que en dicha práctica se está caminando en un nuevo tipo de educación y se están encontrando formas para que el ejercicio de la autonomía pueda ser mucho más efectivo en medio de las condiciones institucionales señaladas anteriormente.

Por otro lado, cabe afirmar que desde la propuesta de FpN una educación debería estar basada en la búsqueda de un pensamiento reflexivo que tenga como principio la autonomía. Deberá ser una educación que no imponga saberes como absolutos, que no condicione la creación de juicios y criterios, que no prescriba nada a los educandos más que nociones e ideas flexibles a cambios y a nuevas razones. La autonomía será, en términos de Kant, un hacer uso público de la razón exponiendo libremente las ideas, es decir, un pensar libre ${ }^{14}$.

Para Lipman, el desarrollo de la autonomía en el marco de un proceso reflexivo debe estar atravesado por lo social y comunitario; la finalidad de tal autonomía debe estar en articular las causas de conflicto en la comunidad y desarrollar los argumentos que apoyan los diversos puntos de vista para finalmente, a partir de la deliberación y el diálogo, lograr una comprensión del panorama que permita realizar un juicio basado en razones sólidas.

\subsection{Diálogo}

Pasemos ahora a considerar la noción de diálogo. Este término viene del griego $\delta i \alpha-\lambda o \gamma o \varsigma$, que significa desenvolvimiento de la palabra. El diálogo se define como una discusión organizada mediante preguntas y respuestas entre individuos interesados por una misma cuestión que se intenta precisar, y respecto de la cual se pueden mantener inicialmente puntos de vista distintos ${ }^{15}$.

El diálogo puede ser tomado como el ejercicio mediante el cual se buscan verdades. Así, para Sócrates las verdades no son establecidas por los sofistas, quienes predicaban un saber definido y acabado, sino que son engendradas mediante un proceso dialógico de preguntas y respuestas. Para Sócrates, este ejercicio debe ser realizado por los filósofos, quienes no deben encargarse de formular respuestas, sino preguntas, puesto que la verdad se construye comunitariamente. Sócrates destaca el valor de la pregunta, justificando que es ella la que abre espacio a la comunicación e instaura el diálogo. La única verdad segura es que no sabemos nada. A partir de la pregunta es como se abre la

\footnotetext{
13 Mónica Monroy y Paola Ramírez. Entrevista a Javier Mendoza, Centro Educativo Amigoniano Escuela de Trabajo El Redentor, 2007.

${ }^{14}$ Es conveniente aclarar que según Lipman, Kant entendía que pensar por sí mismo era la obediencia voluntaria de cada individuo a los principios que pudieran generalizarse como universales. Cfr. Lipman, Op. cit., p. 49.

${ }^{15}$ Martínez y Cortés, Op. cit.
} 
posibilidad del conocimiento y se lanza el pensar hacia un mundo incierto y desconocido que solo a partir de la comunicación se puede comprender.

El diálogo real y verdadero tiene dos aspectos. El primero: el pensamiento de un individuo es siempre incierto; puede enredarse en errores y es solamente mediante la confirmación de otro individuo que nuestro pensamiento se afirma. El segundo aspecto tiene un sentido más profundo: el pensamiento individual sólo puede avanzar en línea recta, sacar conclusiones de lo ya conocido, pero, de hecho, no es productivo. El pensamiento sólo se hace productivo cuando otro individuo irrumpe el discurso, cuando plantea objeciones que lo obligan a uno a repensar.

Este proceso es realizado no solo cuando el uno sólo habla y el otro sólo interrumpe, sino cuando ambos participan alternadamente, hablando e interrumpiendo. Es en este momento cuando comienza el verdadero diálogo. Pero ello es algo diferente de la teoría del consenso, según la cual el criterio de la verdad es el acuerdo con el otro. En nuestro caso no se trata de un criterio para una verdad ya establecida, sino del nacimiento de la verdad misma. Es en este sentido como hablo del diálogo como un momento de la verdad ${ }^{16}$.

El descubrimiento de verdades a partir del diálogo implica repensar el papel del educando y del educador, pues desde el diálogo se deben agudizar las habilidades de razonamiento y de investigación, destacándose un aspecto comunitario y comunicativo, es decir, que tanto educandos como educadores intervengan en la comunicación de manera activa y compartan los logros de su actividad. Lo hallado se debe comunicar a los demás para su discusión ${ }^{17}$.

La verdad más plena es dialogal, esto es, la que se construye de manera activa entre varios sujetos a partir de preguntas y respuestas. Un ejemplo que permite ilustrar esta afirmación es el de Hans-Georg Gadamer, referido en una conferencia en Bogotá.

Una vez, hará ya casi sesenta años, aserramos con Heidegger un tronco en la selva negra, valiéndonos de una sierra de leñador, una de esas sierras que manejaban entre dos, halando el uno de un lado y el otro de otro lado. Cuando cumplí ochenta años reprodujo la prensa la fotografía en la que Heidegger y yo aparecemos aserrando juntos; un periodista me preguntó: ¿cuál de los dos aserraba mejor? Este hombre no entendía nada del asunto.

Cuando se asierra con una de esas sierras no puede haber uno que lo haga mejor que el otro. Lo mismo precisamente ocurre en el hablar con alguien, no puede haber uno que hable mejor que el otro, porque sólo el comprenderse a sí mismo es el sentido del hablar. Sólo al moverse libremente por entre la madera, corta la sierra. Esta es pues la peculiar manera de ser del lenguaje que se forma así en la pregunta y respuesta; es un diálogo sin fin en el que nos movemos como hablantes ${ }^{18}$.

\footnotetext{
${ }^{16}$ Otto F. Bollnov, "La responsabilidad de la razón en el mundo y la sociedad actuales". En: Universitas Philosophicas. Bogotá. N. ${ }^{\circ} 13$; diciembre de 1989. pp. 62-63.

${ }^{17}$ Cfr. Jaime Hoyos, "El seminario en la experiencia docente en la Facultad de Filosofía de la Pontificia Universidad Javeriana”. En: Universitas Philosophica, N. ${ }^{\circ}$ 10. Bogotá, junio 1988. p. 46.

${ }^{18}$ Hans-Gerg Gadamer, "Fenomenología, hermenéutica y la posibilidad de la metafísica". En: Universitas Philosophica, N. ${ }^{\circ}$ 10. Bogotá, junio 1988. pp.46-47.
} 
Por otro lado, las ideas formuladas en un grupo de diálogo no serán consideradas como descabelladas ni absurdas, sino como ideas de seres que piensan y sienten, que tienen la capacidad de razonar y ser razonables.

Los individuos, a partir del diálogo, serán considerados como personas, como sujetos que son en sí mismos fines y no medios o instrumentos para encontrar verdades. La concepción de persona varía a partir del concepto de diálogo, ya que no será un simple objeto como puede haber sido tomado en la educación tradicional en la que muchas veces se ha considerado como tabula rasa ${ }^{19}$ u objeto de experimentación que debe ser llenado de informaciones y conocimientos, sino que se considerará como un sujeto que piensa por sí mismo.

Dicha categoría de diálogo ha logrado desarrollarse en la práctica en El Redentor a través de ejercicios cercanos a las experiencias de vida de los jóvenes participantes, en donde el ejercicio lúdico, la reconstrucción de historias de vida y el elemento estético se han relacionado adecuadamente con las características sociales y culturales de la población involucrada. Una de estas experiencias es citada por una de las practicantes en el diario de campo realizado en el primer semestre de 2006. En él se señalan algunas de las posibilidades de diálogo que se han ido generando en las comunidades de $E l$ Redentor.

Pasamos a preguntar ¿Quién hacía las leyes? En medio de divagaciones dijeron que el Estado, que Dios. Así que preguntamos nuevamente que si sólo esas dos entidades, por medio de reflexiones dijeron que no, que también la sociedad. Y ¿Quién es la sociedad? Dijimos- "eh... pues nosotros", dijo uno de ellos y concluimos que la sociedad somos todos. Luego preguntamos ¿para qué sirven las leyes? El chico nuevo respondió rápidamente que para vivir tranquilos (claro que después aclaró que él no creía en eso), otro dijo que sí, que para vivir bien había que cumplirlas, entonces el anterior chico le preguntó que si creía en eso por qué estaba allí. Esto fue muy interesante porque nos demostró que sí escuchan al otro y además pueden ser capaces de reflexionar sobre lo que ese otro dice, así la comunidad de diálogo empieza a fortalecerse ${ }^{20}$.

Así pues, la práctica educativa orientada desde un modelo reflexivo propugnará el ejercicio del diálogo y la autonomía, como categorías que hacen posible una reflexión más objetiva y un pensar más crítico.

En las prácticas citadas en el centro El Redentor podría identificarse a partir de lo anterior que el diálogo más importante lo constituyó el proyecto mismo de $\mathrm{FpN}$ con el modelo pedagógico y terapéutico de la institución, para lograr identificar los caminos posibles para desarrollar la comunidad de indagación. En esta medida, dicha práctica permitió reafirmar la comunidad de indagación como un espacio oportuno y preciso que permite la construcción de un pensamiento crítico y creativo dado en el intercambio de razones y opiniones en torno a una práctica dialógica y respetuosa. Javier Mendoza, coordinador académico del centro El Redentor afirma al respecto: "La comunidad de indagación le permitió a los muchachos resignificar su convivencia en un momento

${ }^{19}$ Tabula rasa, para Aristóteles significa «una tablilla en la que no hay nada escrito». Para los empiristas ingleses esta idea fue utilizada para negar cualquier posibilidad de ideas innatas y afirmar taxativamente que el conocimiento empieza por los sentidos. Diccionario de filosofía. Editorial Herder, Barcelona, 2000 .

${ }^{20}$ Diana León, Diario de campo N. ${ }^{\circ}$ 04, Práctica profesional II. Primer semestre de 2006. 
determinado [...] es bien interesante el hecho de que los muchachos lograron ajustar su comportamiento dialogando a través de las comunidades de indagación"21.

Algo que llamó la atención fue el cambio de comportamiento solamente dialogando con ellos y haciendo todo ese compartir; ese reconocimiento logró un cambio de comportamiento en los muchachos, entonces si nosotros trabajamos con menores infractores, con problemas de conducta, por llamarlo de otra manera, podríamos decir que el programa de $\mathrm{FpN}$ a través de sus técnicas, porque cuando hablo de comunidades de indagación podría pensarse en una técnica, me refiero mejor a FpN desarrollada en jóvenes con problemas de conducta que opera de una manera significativa y positiva en el pensamiento, lo que se traduce posteriormente en cambios de su comportamiento y su repertorio conductual para también llamarlo de otra manera ${ }^{22}$.

Así pues, la práctica de filosofía para niños en este centro reeducativo ha permitido ampliar el horizonte de comprensión de un proyecto de educación filosófica que va más allá de las aulas tradicionales y de las prácticas escolares comunes. Dicho proyecto puede, a través del ejercicio del pensamiento autónomo, lograr generar dentro de la comunidad espacios de diálogo y reflexión, que a su vez les permiten a los jóvenes descubrir nuevas formas de ver el mundo y de participar en él.

Finalmente, buscando concluir esta reflexión dando voz a quienes han venido revisando la relación entre FpN y la educación social, quisiera citar un aparte del estudio realizado por las estudiantes Paola Ramírez y Mónica Monroy, con quienes logramos adelantar un ejercicio consciente y delicado de sistematización sobre la práctica de FpN en el centro El Redentor:

Ahora bien, la comunidad de indagación se convirtió en el centro El Redentor en el medio por el cual los muchachos encontraban un espacio no sólo para el desarrollo de aquellas habilidades propuestas por el programa, sino a su vez, en el lugar, para compartir y para expresar aquellas dudas que surgen al interactuar con otros de manera crítica y reflexiva. Además, la comunidad de indagación tomó un papel importante en el reconocimiento de los otros, ya que permitió que a partir del proceso dialógico que surge en la comunidad, se le diera importancia al aporte que los otros pueden ofrecer a partir de sus experiencias de vida para la construcción del pensamiento.

Queda visto cómo filosofía para niños, en sectores sociales marginados y en comunidades con características tan particulares como las del Centro El Redentor, ayuda no solo en la construcción y en la resignificación del pensamiento, sino también en la elaboración de una comunidad en donde lo que prima es el reconocimiento por la individualidad de cada uno de sus miembros y en la posibilidad de que cada uno pueda transformar su entorno a partir de la acción que se emprende en comunidad. Ahora bien, el que FpN pueda ofrecerle a una institución como lo es el Centro El Redentor nuevas estrategias para la ejecución y para el fortalecimiento de un programa reeducativo permite ampliar el horizonte de comprensión de esta propuesta que desde la filosofía genera transformación social e impulsar un trabajo serio enfocado hacia el planteamiento de nuevos fundamentos filosóficos y hacia la construcción de recursos didácticos que garanticen la buena práctica de este proyecto.

\footnotetext{
${ }^{21}$ Monroy y Ramírez, Op. cit.

${ }^{22}$ Ibídem.
} 
Como aquí se señala, la práctica de filosofía para niños en El Redentor, institución que atiende particularmente a una población marginada, en el sentido de personas que han cometido acciones delictivas y que están cumpliendo una sanción privadas de la libertad, ha logrado identificar un camino certero para el ejercicio del diálogo y para el desarrollo de un pensamiento autónomo. La práctica de la comunidad de indagación ha sido el recurso pedagógico desde el cual se ha logrado dicho avance y ha iniciado la construcción de estrategias didácticas que basadas en una rigurosa reflexión filosófica podrán continuar aportando en la formación de personas críticas en nuestra sociedad.

\section{Bibliografía}

BollnOV, Otto F. "La responsabilidad de la razón en el mundo y la sociedad actuales". Universitas Philosophicas n. ${ }^{\circ}$ 13, diciembre de 1989, pp. 62-63. Bogotá.

GADAMER, Hans-Gerg. "Fenomenología, hermenéutica y la posibilidad de la metafísica”. Universitas Philosophica, n. ${ }^{\circ}$ 10, junio 1988, pp. 46-47. Bogotá.

Hoyos, Jaime. "El seminario en la experiencia docente en la Facultad de Filosofía de la Pontificia Universidad Javeriana". Universitas Philosophica, n. ${ }^{\circ}$ 10, junio 1988, p. 46. Bogotá.

KANT, Emmanuel. Respuesta a la pregunta ¿Qué es la Ilustración?, en Filosofía de la historia. Buenos Aires: Nova, 1064.

LEÓn, Diana. Diario de campo N. ${ }^{\circ}$ 04, Práctica profesional II. Primer semestre de 2006.

LiPMAn, Matthew. Pensamiento complejo y educación. Madrid: Editorial De la Torre, 1997.

Malagón, Edwin. Diario de campo 02. Práctica profesional II. 2006.

MARTínez Riu, Antoni y Cortés Morató, Jordi. Diccionario de Filosofía. Barcelona: Editorial Herder, CD-ROM, 1998.

Monroy, Mónica y Ramírez, Paola. Entrevista a Javier Mendoza, Centro Educativo Amigoniano, Escuela de trabajo El Redentor. 2007.

PAI. Proyecto de Atención Institucional - Centro Educativo Amigoniano: Escuela de Trabajo El Redentor. 2001-2003.

RoJAS, Víctor Andrés. Entrevista: Lida Gil. Corporación Universitaria Minuto de Dios. 2007.

SHARP, Ann Margaret. "La comunidad de cuestionamiento e investigación filosóficos: Educación para la democracia”. En: ¿Qué es Filosofía para Niños? Ideas y propuestas para pensar la educación. Buenos Aires: Editorial Universidad de Buenos Aires, pp.177, 1997.

VILlamil, Nubia. Diario de campo 03. Práctica Profesional III. 2006. 